Chronic Obstructive Pulmonary Diseases:

Journal of the COPD Foundation

\title{
Editorial
}

\section{What's New with the St George's Respiratory Questionnaire and Why Do We Care?}

\author{
Megan Hardin, MD, MPH ${ }^{1}$ Steven I. Rennard, $\mathrm{MD}^{2,3}$
}

\begin{abstract}
Abbreviations: chronic obstructive pulmonary disease, COPD; COPD Biomarkers Qualification Consortium, CBQC; St George's Respiratory Questionnaire, SGRQ; body mass index, BMI; socioeconomic status, SES; minimum clinically important difference, MCID

Citation: Hardin M, Rennard SI. What's new with the St George's Respiratory Questionnaire and why do we care? Chronic Obstr Pulm Dis. 2017;4(2):83-86. doi: https://doi.org/10.15326/jcopdf.4.2.2017.0139
\end{abstract}

1 Clinical Discovery Unit, Early Clinical Discovery, AstraZeneca, Waltham, Massachusetts

2 Clinical Discovery Unit, Early Clinical Discovery, AstraZeneca, Cambridge, United Kingdom

3 University of Nebraska Medical Center, Omaha

\section{Address correspondence to:}

Megan Hardin, MD, MPH

Clinical Discovery Unit

Early Clinical Discovery

AstraZeneca

Waltham, MA

email: megan.hardin@astrazeneca.com

\section{Keywords:}

chronic obstructive pulmonary disease; COPD; St George's Respiratory Questionnaire; SGRQ; COPD Biomarkers Qualification Consortium; CBQC

This issue of the Journal includes 5 companion papers ${ }^{1-5}$ together with a supplemental set of data files and an introduction ${ }^{6}$ from the Chronic Obstructive Pulmonary Disease (COPD) Biomarkers Qualification Consortium (CBQC). ${ }^{7}$ This body of work summarizes the evaluations performed to establish the St George's Respiratory Questionnaire (SGRQ) as a qualified drug development tool. ${ }^{8}$ It contributes to our field in 3 major ways that this editorial will briefly address. First, while the SGRQ has been very widely used since it's description ${ }^{9}$ in 1992 with 1057 citations listed in Medline (accessed Feb 2017), the analyses provided in the accompanying papers have further added to our understanding of how the SGRQ performs. Second, despite its wide use in clinical trials, qualification of the SGRQ will be important in the development of new treatments for COPD. Finally, the process by which these studies were conducted by the $\mathrm{CBQ} \mathrm{C}^{10}$ represents a relatively new model of collaboration, addressing questions that have been difficult to approach using traditional research structures. Similar approaches may be increasingly important as our understanding of COPD advances.

The accompanying papers included data from 17 interventional studies with 26857 participants and 3 observational studies with 7500 participants. Follow up across the studies varied from less than 1 year to 4 years. The studies were not a random sample but were selected based on size, duration, anticipated data quality and content and geographic distribution. The size and composition of the resulting analysis helps inform several questions that have been modestly controversial. For example, baseline SGRQ was not significantly related to smoking status, gender or age, ${ }^{4}$ although these have been reported in some but not all smaller studies. ${ }^{11,12}$ In contrast, as expected, the SGRQ score was related to features of the disease including dyspnea, lung function, comorbidity and body mass index (BMI). ${ }^{4}$ Interestingly, baseline SGRQ was related to socioeconomic status (SES) in observational studies, although the effect was smaller in interventional studies.

The response of the SGRQ was also related to SES. The SGRQ regularly shows a clear Hawthorne effect; namely, an improvement is observed among participants in clinical trials assigned to placebo groups. ${ }^{13}$ In low SES countries there was a greater response among participants assigned to placebo by 
about 2 units compared to high SES countries. The overall difference from before to after in the SGRQ among low SES countries sometimes exceeded the minimum clinically important difference (MCID) ${ }^{7}$ by 4 units. ${ }^{1}$ The mechanisms accounting for the Hawthorne effect have been much debated but likely include better access to health care and improved compliance while in clinical trials. The observations from the paper by Jones et al suggest that these effects may be greater in low SES countries. However, these effects do not invalidate studies that pool data across countries with varying SES to assess drug effect as the differences between active treatment and placebo were the same in high and low SES countries. That is, the SGRQ can detect differences when participants from countries of high and low SES enter clinical trials and are treated with a placebo. The SGRQ can also detect differences between treatment and placebo in both high and low SES countries that are similar in size and are superimposed on the Hawthorne effect. This is an important finding that assures regulators that the SGRQ is a qualified decision-making tool that can be used with confidence across a variety of populations including those with varied SES.

The SGRQ is a scale with Rasch modelling characteristics. ${ }^{9}$ That is, it functions as a linear ruler, and a difference at the top of the scale has the same significance as the same difference at the bottom. This is essential for comparing average differences among individuals with different absolute scores. Having Rasch characteristics, however, does not mean that severity does not affect how patients respond. In this context, the analysis by Müllerova et al confirms that baseline SGRQ status predicts exacerbation, hospitalization and mortality risk. ${ }^{3}$ Baseline health status also affected the SGRQ response to intervention. Specifically, those with less dyspnea or better lung function responded with greater improvement in SGRQ when given treatments. ${ }^{3}$ This finding will help the interpretation of results of trials conducted in groups with differing severity. Importantly, this finding contributes to the growing body of information that patients with milder disease may be at substantial risk and may be those for whom therapy may have the greatest benefit. ${ }^{14}$

The papers in the current issue also contribute to our understanding of how the SGRQ should be interpreted and suggest how it can be used more effectively in the future. In assessing any test, it is important to define the MCID. ${ }^{7}$ This is the difference that would generally be recognized by a patient or clinician as being meaningful.
Differences smaller than this are often regarded as clinically unimportant even if statistically significant. However, the MCID is most commonly determined in a population that may include a number of individuals with large responses and others with no response (or worsening). Thus, basing clinical importance on an average MCID may miss the presence of a substantial responding population.

A responder analysis, which determines the number of individuals who obtain a satisfactory response, is a much more robust way of assessing interventions. ${ }^{15}$ This approach has been instrumental in the substantial advances in cancer chemotherapy in recent decades and has the potential for similar impact among COPD subsets. Responder analysis, however, requires a robust definition of who is a responder. In this regard, the CBQC analyses have provided crucial information demonstrating that responder analysis can determine differences between effective treatment and placebo over a range of MCIDs. ${ }^{2}$ The conventionally-used 4 unit difference performed very well. Interestingly, thresholds above and below 4 units also distinguished between effective treatment and placebo. These findings do not change the definition of clinically important, but they can have important implications for drug development strategy. This is particularly important as responder analyses can be used to identify subsets appropriate for specific interventions, an approach that will become increasingly important with the development of personalized medicines that are mechanistically based.

The SGRQ has been widely used in many settings, including drug development. Findings based on the SGRQ have been incorporated into drug labels. Nevertheless, qualification and the recent incorporation of the SGRQ into the Food and Drug Administration's draft guidance is an important step forward. ${ }^{8}$ Current drug development can cost in excess of $\$ 2.5$ billion. ${ }^{16}$ Qualification of a measure allows it to be used with confidence that it is acceptable in the qualified context of use for regulatory purposes. Absent qualification, regulatory agencies can question the validity of the measure even if a significant result is obtained in a pivotal clinical trial. This uncertainty can have a very negative effect on development of new treatments. Specifically, facing uncertainty, sponsors may choose to invest the substantial resources required to bring a new medicine to the clinic in therapeutic areas with more established regulatory paths.

Despite the substantial advances in understanding 
COPD pathogenesis and pathophysiology, only 1 new class of drug has been introduced in the last 30 years. ${ }^{17}$ The incorporation of the SGRQ into the Draft Guidance is a step that will help advance the field. ${ }^{8}$ However, it is certain that additional drug development tools will be required to properly assess the many promising approaches currently being investigated. The CBQC is one of many academic/industry/government collaborations designed to advance the development of new medications. It has also highlighted several issues inherent in such efforts.

The data on which the CBQC analyses of the SGRQ were based were generated as part of clinical trials that cost considerably more than a conventional drug development program. The data were available because the sponsors of the component studies, which were performed for other purposes, were willing to share data at no cost. The majority of the data came from industry sources that have generally regarded the development of drug development tools as pre-competitive areas where cooperation is best for the entire field. Importantly, several industry participants also contributed financial support required for analyses. The formation of the CBQC was based on this cooperative model as well as the explicit policy that all findings would be publicly available and that use of any tools qualified would not be restricted by any activities of the CBQC. ${ }^{10}$ Academic institutions also contributed data from studies funded by the government with the same principles of broad and free sharing of findings.

Several issues arose during the analysis process. Industry collaborators were unwilling to share individual participant level data with drug specific information with their competitors. As a result, third parties were selected to aggregate and analyze data and, therefore, treatment response is limited to drug class. Some potential industry collaborators felt it inappropriate to support an activity that would benefit their competitors even if they did not contribute. The degree to which this limited participation in the Consortium is unclear, but it is a realistic concern. Similarly, several potential academic collaborators were unable to resolve issues related to data ownership and intellectual property. This is an interesting paradox when for profit entities are acting in an altruistic manner and non-profit groups are concerned with asset preservation. However, the concerns are understandable as universities try to meet difficult financial demands by exploiting their intellectual assets.

In summary, the papers in this issue of Chronic Obstructive Pulmonary Diseases: Journal of the COPD Foundation advance our understanding of the SGRQ. They have resulted in the SGRQ becoming a drug development tool that can be used with confidence. Without doubt, the SGRQ is just one of many new drug development tools that will be needed to advance the treatment of COPD. The CBQC achieved this goal through pooling of data and free sharing of results, providing an example process for how to address challenges that have plagued our field going forward.

\section{Declaration of Interest}

$\mathrm{MH}$ is an employee of AstraZeneca; SIR is an employee of AstraZeneca and holds shares in the company as part of his employment. In the last year, he has received an honorarium from the Japanese Respiratory Society. 


\section{References}

1. Jones PW, Gelhorn H, Wilson H, et al. Socioeconomic status as a determinant of health status treatment response in COPD trials. Chronic Obstr Pulm Dis. 2017; 4(2): In press.

doi: https://doi.org/10.15326/jcopdf.4.2.2017.0132

2. Jones PW, Gelhorn H, Wilson H, et al. Responder analyses for treatment effects in COPD using the St George's Respiratory Questionnaire. Chronic Obstr Pulm Dis. 2017; 4(2): In press. doi: https://doi.org/10.15326/jcopdf.4.2.2017.0130

3. Müllerova H, Gelron H, Wilson H, et al. St. Georges Respiratory Questionnaire score predicts outcomes in patients COPD: Analysis of individual patient in the COPD Biomarkers Qualification Consortium database. Chronic Obstr Pulm Dis. 2017; 4(2): In press.

doi: https://doi.org/10.15326/jcopdf.4.2.2017.0131

4. Tabberer M, Benson VS, Gelhorn H, et al. The COPD Biomarkers Qualification Consortium database: Baseline characteristics of the St George's Respiratory Questionnaire dataset. Chronic Obstr Pulm Dis. 2017; 4(2): In press.

doi: https://doi.org/10.15326/jcopdf.4.2.2017.0128

5. Jones PW, Gelhorn H, Karlsson N, et al. Baseline as predictor of change in St George's Respiratory Questionnaire score in trials of long-acting bronchodilators with COPD patients. Chronic Obstr Pulm Dis. 2017; 4(2): In press.

doi: https://doi.org/10.15326/jcopdf.4.2.2017.0139

6. Tabberer M, Jones PW. The COPD Biomarkers Qualification Consortium St George's Respiratory Questionnaire: Output of a consortium to advance drug development. Chronic Obstr Pulm Dis. 2017; 4(2): In press.

doi: https://doi.org/10.15326/jcopdf.4.2.2017.0127

7. Rennard SI. Minimal clinically important difference, clinical perspective: An opinion. COPD. 2005; 2(1): 51-55. doi: https://doi.org/10.1081/COPD-200050641

8. U.S. Food and Drug Administration (FDA). Chronic obstructive pulmonary disease: Developing drugs for treatment. Guidance for industry. FDA website. https://www.fda.gov/downloads/ drugs/guidances/ucm071575.pdf

Published May 2016. Accessed February 2017.

9. Jones PW, Quirk FH, Baveystock CM, Littlejohns P. A selfcomplete measure of health status for chronic airflow limitation. The St George's Respiratory Questionnaire. Am Rev Respir Dis. 1992;145(6):1321-1327.

doi: https://doi.org/10.1164/ajrccm/145.6.1321

10. Casaburi R, Celli B, Crapo J, et al. The COPD Biomarker Qualification Consortium (CBQC). COPD. 2013;10(3): 367-377. doi: https://doi.org/10.3109/15412555.2012.752807

11. de Torres JP, Casanova C, Hernandez C, Abreu J, AguirreJaime A, Celli BR. Gender and COPD in patients attending a pulmonary clinic. Chest. 2005; 128(4):2012-2016. doi: https://doi.org/10.1378/chest.128.4.2012
12. Miravitlles M, Ferrer M, Pont A, et al. Characteristics of a population of COPD patients identified from a populationbased study. Focus on previous diagnosis and never smokers. Respir Med. 2005; 99(8):985-995. doi: https://doi.org/10.1016/j. rmed.2005.01.012

13. Jones PW, Bech KM, Chapman KR, Decramer M, Mahler DA, Wedzicha JA. Minimal clinically important differences in pharmacological trials. Am J Respir Crit Care Med. 2014. 189(3): 250-255. doi: https://doi.org/10.1164/rccm.201310-1863PP

14. Woodruff PG, Barr G, Bleecker E, et al. Clinical significance of symptoms in smokers with preserved pulmonary function. $N$ Engl J Med. 2016; 374(19):1811-1821.

doi: https://doi.org/10.1056/NEJMoa 1505971

15. Jones PW, Rennard S, Tabberer M, Riley JH, Vahdati-Bolouri $\mathrm{M}$, Barnes NC. Interpreting patient-reported outcomes from clinical trials in COPD: A discussion. Int J Chron Obstruct Pulmon Dis. 2016;11: 3069-3078. doi: https://doi.org/10.2147/COPD.S117378

16. DiMasi JA, Grabowski HG, Hansen RW. Innovation in the pharmaceutical industry: New estimates of R\&D costs. J Health Econ. 2016; 47:20-33. doi: https://doi.org/10.1016/j.jhealeco.2016.01.012

17. Tashkin DP. Roflumilast: The new orally active, selective phophodiesterase-4 inhibitor, for the treatment of COPD. Expert Opin Pharmacother. 2014; 15(1): 85-96. doi: https://doi.org/10.1517/14656566.2013.837159 